\title{
EVALUACIÓN DEL CONOCIMIENTO BÁSICO EN QUÍMICA EN ESTUDIANTES DE EDUCACIÓN DIVERSIFICADA: EL CASO DE INGENIERÍA INDUSTRIAL, UNED.
}

\section{EVALUATION OF BASIC KNOWLEDGE IN CHEMISTRY DIVERSIFIED EDUCATION STUDENTS: THE CASE OF INDUSTRIAL ENGINEERING, UNED.}

\section{Rodolfo Hernández-Chaverri', rohernandez@uned.ac.cr Eric Montero-Miranda'2. emonterom@uned.ac.cr Wendy Villalobos-González ${ }^{3}$, wvillalobosg@uned.ac.cr Universidad Estatal a Distancia, Costa Rica}

\author{
Volumen 8, Número 2
}

30 de noviembre de 2017

pp. $1-23$

Recibido: 28 de marzo de 2017

Aprobado: 07 de setiembre de 2017

\footnotetext{
${ }^{1}$ Ingeniero Químico, Universidad de Costa Rica, Master en Sistemas Modernos de Manufactura, Instituto Tecnológico de Costa Rica. Director de la Cátedra de Ciencias Químicas para ingeniería, Universidad Estatal a Distancia, Costa Rica. Correo electrónico: rohernandez@uned.ac.cr

${ }^{2}$ Químico Industrial, Universidad Nacional, Profesor tutor del Departamento de Ciencias Químicas de la Universidad Estatal a Distancia, Costa Rica. Correo electrónico: emonterom@uned.ac.cr

${ }^{3}$ Química Industrial, Universidad Nacional, Directora da la Cátedra de Ciencias Químicas, Universidad Estatal a Distancia, Costa Rica. Correo electrónico: wvillalobosg@uned.ac.cr
}

Evaluación del conocimiento básico en química en estudiantes de educación diversificada: El caso de Ingeniería Industrial, UNED. 


\section{Resumen}

Uno de los grandes retos que tiene el Ministerio de Educación Pública (MEP) en Costa Rica es mejorar la calidad en la enseñanza de la química a nivel de secundaria y disminuir la brecha entre colegios de enfoque científico y académico. La Universidad Estatal a Distancia (UNED), como un ente de educación superior preocupado por esta problemática, creó un plan diagnóstico-nivelatorio en química para establecer conocimientos básicos de esta materia para los estudiantes que ingresan a la carrera de Ingeniería Industrial, dicho plan consistió en dos etapas: La primera etapa, se llevó a cabo entre los años 2013 y 2014, cuando se realizó una prueba diagnóstica, misma que fue aplicada a colegios científicos y académicos que cursaban el ciclo diversificado y a los estudiantes que deseaban ingresar a la carrera de ingeniería industrial. La segunda etapa, consistió en crear un curso nivelatorio en química, con el fin de que los estudiantes de primer ingreso a carrera adquiriesen el conocimiento básico para afrontar cursos universitarios de Química General I, mismo que se ha impartido desde el año 2014 hasta la fecha por la UNED. Este artículo presenta la experiencia de la Cátedra de Ciencias Químicas en el diseño y aplicación de la prueba diagnóstica y los primeros resultados que se desprenden del curso nivelatorio de química, como una estrategia para mejorar los rendimientos académicos de los estudiantes que ingresan a la UNED.

Palabras clave: prueba diagnóstica; Química Nivelatoria; mejoramiento de la calidad de la enseñanza; rendimiento académico; Ingeniería Industrial.

\section{Abstract}

One of the great challenges that the Ministry of Public Education (MEP) has in Costa Rica is to improve the quality of teaching chemistry at the secondary level and reduce the gap between scientific and academic schools. The Universidad Estatal a Distancia (UNED), as a higher education institution worried about this problem created a diagnostic-level plan in chemistry to establish basic knowledge of this subject for students entering the Industrial Engineering career, this plan consisted of Two stages: The first stage was carried out between 2013 and 2014 where a diagnostic test was carried out, which was applied to scientific and academic colleges that attended the diversified cycle and to students who wanted to enter the industrial engineering career. The second stage was to create a leveler course in chemistry, in order for first-year students to acquire basic knowledge to undertake university courses in General Chemistry I, which has been taught since 2014 to date by the UNED. This paper presents the experience of the Chair of Chemical Sciences in the design and implementation of the diagnostic test and the first results obtained from the leveling chemistry course as a strategy to improve the academic performance of students entering the UNED.

Keywords: diagnostic test; Chemistry Leveler; improving the quality of teaching; academic performance; Industrial Engineering.

Evaluación del conocimiento básico en química en estudiantes de educación diversificada: El caso de Ingeniería Industrial, UNED. 


\section{INTRODUCCIÓN}

La educación es una herramienta importante que facilita el acceso a nuevas oportunidades, y con ello obtener una mejora en la calidad de vida. Las instituciones de educación cumplen un papel importante dentro de la sociedad, ya que son las encargadas de preparar y formar los futuros profesionales de la misma.

En Costa Rica, mediante la Ley № 2160 sobre la Ley Fundamental de la Educación, en su artículo 1 estipula que toda persona tiene derecho a la educación y que el Estado será quien les facilite este derecho. Por otro lado, en su artículo 14 inciso f), referido a la educación media; uno de los objetivos para atender las necesidades vocacionales de los estudiantes es el de promover el desarrollo de las habilidades y aptitudes que le permitan al estudiante orientarse hacia algún campo de actividades vocacionales o profesionales.

Teniendo en cuenta este antecedente de la ley costarricense, los centros de educación media deben de tener la capacidad de solventar las necesidades que los y las adolescentes requieran para su futuro ingreso a una carrera a nivel universitario. En algunos casos esta función se cumple, sin embargo, no es la regla general en la mayoría de los centros educativos del país. La falta de recursos que le permitan al docente desarrollar y brindar una clase de calidad para el estudiante, es una limitante muy común en las aulas.

Otro problema es el que destaca Sol (2004), en donde se enfatiza el tema de la falta de capacitación docente por parte del Estado, en este diagnóstico Sol indica que la problemática existe desde los años 1970 y como se pone en evidencia la carencia de coordinación, concertación y fiscalización en la formación docente por parte de los últimos gobiernos. Es evidente que esta falta de formación y actualización de los conocimientos docentes, genera la problemática a la hora de pasar estos conocimientos al estudiante.

Evaluación del conocimiento básico en química en estudiantes de educación diversificada: El caso de Ingeniería Industrial, UNED. Rodolfo Hernández-Chaverrí, Eric Montero-Miranda

Wendy Villalobos-González.

DOI : http://dx.doi.org/10.22458/caes.v8i1.1915 
Actualmente, nuestro país cuenta con pocas instituciones que brindan esa actualización, que el docente requiere, básicamente estas entidades se limitan al Ministerio de Educación Pública (MEP) y las universidades estatales, quienes realizan capacitaciones anuales en temas específicos. Igualmente, está la Fundación para el Centro Nacional de la Ciencia y la Tecnología (CIENTEC) que es una organización no gubernamental creada para promover y popularizar la ciencia y la tecnología en el país (Ramírez, 2006).

Uno de los grandes retos que ha tenido la educación a nivel nacional, es el mejoramiento de la calidad en la enseñanza de la química a nivel de secundaria, y, sobre todo en los ciclos de educación diversificada. El sistema actual aun cuenta con grandes problemáticas para el desarrollo de las temáticas relacionadas con las ciencias exactas en general, pero en un mayor grado asociado a la química, y estas deficiencias son extendidas también al plano universitario, y esto se evidencia sobre todo en las carreras que incluyen a la química como una base importante de su formación.

La Universidad Estatal a Distancia (UNED), como una estrategia para el mejoramiento de los rendimientos académicos de los estudiantes que ingresan a sus carreras, estableció para el año 2013 un plan piloto para ayudar a aquellos estudiantes que tenían deficiencias en esta ciencia, mediante la implementación de una prueba diagnóstica que incluye las temáticas que el Ministerio de Educación Pública (MEP) utiliza para elaborar las pruebas de bachillerato a nivel nacional. Este examen diagnóstico se validó inicialmente con estudiantes de los colegios científicos costarricenses en la zona de Limón y Alajuela, para luego ser aplicado por primera vez a los estudiantes que optaban por el ingreso a la carrera de Ingeniería Industrial. La validación de estas pruebas en los colegios científicos se realizó partiendo de que en ellos se imparte una formación en química de mayor nivel.

Evaluación del conocimiento básico en química en estudiantes de educación diversificada: El caso de Ingeniería Industrial, UNED. Rodolfo Hernández-Chaverrí, Eric Montero-Miranda

Wendy Villalobos-González.

DOI : http://dx.doi.org/10.22458/caes.v8i1.1915 
En general los colegios científicos costarricenses, desde su creación forman parte de la Política Nacional de Desarrollo Científico y Tecnológico, y fueron creados mediante la Ley № 7169, del 1 de agosto de 1990, con el objetivo principal de brindar una formación integral a sus estudiantes, con énfasis en la adquisición de conocimientos sólidos y habilidades en los fundamentos de la matemática, la física, la química, la biología y la informática, por lo que estos colegios son considerados como una opción eficaz para el mejoramiento de la enseñanza de las ciencias. Estos dedican más tiempo en lecciones en el área de las ciencias exactas, manejando niveles de dificultad muy similares a los universitarios, ya que, en su mayoría, los profesores que imparten lecciones en estos centros son profesores universitarios. Además, el proceso que el colegio aplica para seleccionar sus estudiantes es muy riguroso, en donde se toman los promedios por encima de 85 en los niveles de $7^{\circ}$, $8^{\circ}$ y $9^{\circ}$, y el no haber aplazado nunca una materia, lo que hace que estos colegios seleccionen los estudiantes más sobresalientes del país (C.C.C. San Pedro, 2014).

Este mecanismo utilizado por la UNED y que se ha elaborado en la Cátedra de Ciencias Químicas, pretende exponer un panorama más claro de la realidad existente en cuanto a los niveles académicos en química de los estudiantes costarricenses que ingresan no solo a esta institución, sino a cualquier otra institución de educación superior.

De manera adyacente a esta evaluación, y como una medida para mejorar la calidad académica de los estudiantes que ingresan a esta carrera, la Cátedra de Ciencias Químicas en conjunto con la coordinación de la carrera de Ingeniería Industrial implementan una nueva estrategia para preparar a los estudiantes para los cursos futuros que enfrentarían en su carrera. Con ello se crea el curso de Química Nivelatoria; el cual, tiene por objetivo preparar a los estudiantes en el área de química antes que ingresen a su primer curso de química a nivel universitario. Además, que disminuye la brecha existente entre el ambiente

Evaluación del conocimiento básico en química en estudiantes de educación diversificada: El caso de Ingeniería Industrial, UNED. Rodolfo Hernández-Chaverrí, Eric Montero-Miranda

Wendy Villalobos-González

DOI : http://dx.doi.org/10.22458/caes.v8i1.1915 
de secundaria y universidad; este curso se imparte bajo la modalidad a distancia con tutorías presenciales quincenales y con una duración semestral.

\section{METODOLOGÍA}

La primera parte de este proyecto se llevó a cabo durante los años 2013-2014, e incluye la elaboración de una prueba de ubicación diagnostica con base en el temario de Química del MEP, por lo que se asignó el diseño de ítems de selección única a especialistas en contenidos en química, luego estos fueron evaluados por el departamento de Asesoría Académica de la Escuela de Ciencias Exactas y Naturales de la UNED y un grupo de expertos en Química de la misma institución.

Una vez concretados estos procesos de confección y validación, se procedió a aplicar la prueba diagnóstica que constaba de 70 ítems, a estudiantes de los colegios científicos costarricenses de Alajuela y Limón para el año 2013, y a algunos colegios académicos (modalidad diurna y nocturna) para el año 2014. Esto se realizó con el fin de identificar falencias en conocimientos básicos en química entre ambos tipos de colegios y entrelazar similitudes con los estudiantes aspirantes a la carrera de Ingeniería Industrial.

La prueba diagnóstica fue aplicada a los estudiantes aspirantes a la carrera de Ingeniería Industrial en las zonas de Alajuela y San Carlos para el año 2013 y en las zonas de Alajuela y Palmares en el año 2014. Mediante un tratamiento estadístico descriptivo, se analizaron los datos y se compararon los rendimientos en ambos grupos de estudiantes evaluados para cada año.

La segunda fase del proyecto implicó la elaboración del curso de Química Nivelatoria. Este curso se diseñó con la idea de mejorar el nivel de conocimiento básico en química de los estudiantes aspirantes a la carrera de Ingeniería Industrial de la UNED, con el fin de romper

Evaluación del conocimiento básico en química en estudiantes de educación diversificada: El caso de Ingeniería Industrial, UNED. Rodolfo Hernández-Chaverrí, Eric Montero-Miranda

Wendy Villalobos-González

DOI : http://dx.doi.org/10.22458/caes.v8i1.1915 
esa barrera de transición colegio-universidad, o bien para minimizar esa deficiencia en la materia. El curso es una mezcla de temas básicos de secundaria (según el MEP), con otros de niveles universitarios y se diseñó con plataforma MOODLE.

El curso fue impartido a 120 estudiantes durante el I semestre 2014 y a 63 estudiantes durante el II semestre 2014. Entre el primer y segundo semestre se hizo una modificación en el modelo evaluativo y se compararon los resultados obtenidos en ambos casos, y se determinó cuál generó un mejor rendimiento académico. Desde el II semestre 2014, el curso se sigue impartiendo con el mismo modelo de evaluación.

Una vez que los estudiantes llevaron el curso de Química Nivelatoria el primer y segundo semestre de 2014, se recopilaron los datos de las notas finales del curso para evaluar el rendimiento del plan piloto en la población que realizó el curso en el primer y segundo semestre de 2014. Dichas notas se obtuvieron a partir de las evaluaciones aplicadas en el curso, como exámenes, pruebas cortas en línea y otras actividades por medio de la plataforma.

Para finalizar, en este documento se incluye una comparación entre el rendimiento académico de los estudiantes que cursan el curso de Química I (cuatrimestral) de la UNED y los estudiantes de ingeniería industrial, con el fin de determinar si el curso de Química Nivelatoria ayuda a mejorar el nivel de aprobación del curso de Química I.

\section{RESULTADOS Y ANALISIS}

\subsection{Aplicación de la prueba diagnóstica a estudiantes de secundaria}

Luego de la confección y validación de ítems por parte de los jueces, se realizaron las correcciones necesarias de redacción e interpretación a la prueba definitiva por aplicar a la

Evaluación del conocimiento básico en química en estudiantes de educación diversificada: El caso de Ingeniería Industrial, UNED. Rodolfo Hernández-Chaverrí, Eric Montero-Miranda

Wendy Villalobos-González.

DOI : http://dx.doi.org/10.22458/caes.v8i1.1915 
población meta, la cual se destinó en primera instancia a los estudiantes de colegios científicos costarricenses de la zona de Alajuela y Limón. Como parte de esta experiencia se obtuvieron los resultados que se exhiben a continuación

La prueba para el año 2013 la realizaron un total de 31 estudiantes de ambos colegios científicos (Alajuela y Limón) en donde se observa que la media aritmética en la calificación es de: 79,66 . Por otro lado, en la figura 1 , se observa que la mayoría de estudiantes se encuentran en el rango de notas entre 70 y 80 , lo que representa cerca del $84 \%$ de los estudiantes que realizaron la prueba. El gráfico en sí presenta una distribución normal de los valores obtenidos.

Figura 1. Distribución del porcentaje de estudiantes de Colegios Científicos, según los rangos de calificaciones obtenidos, año 2013.

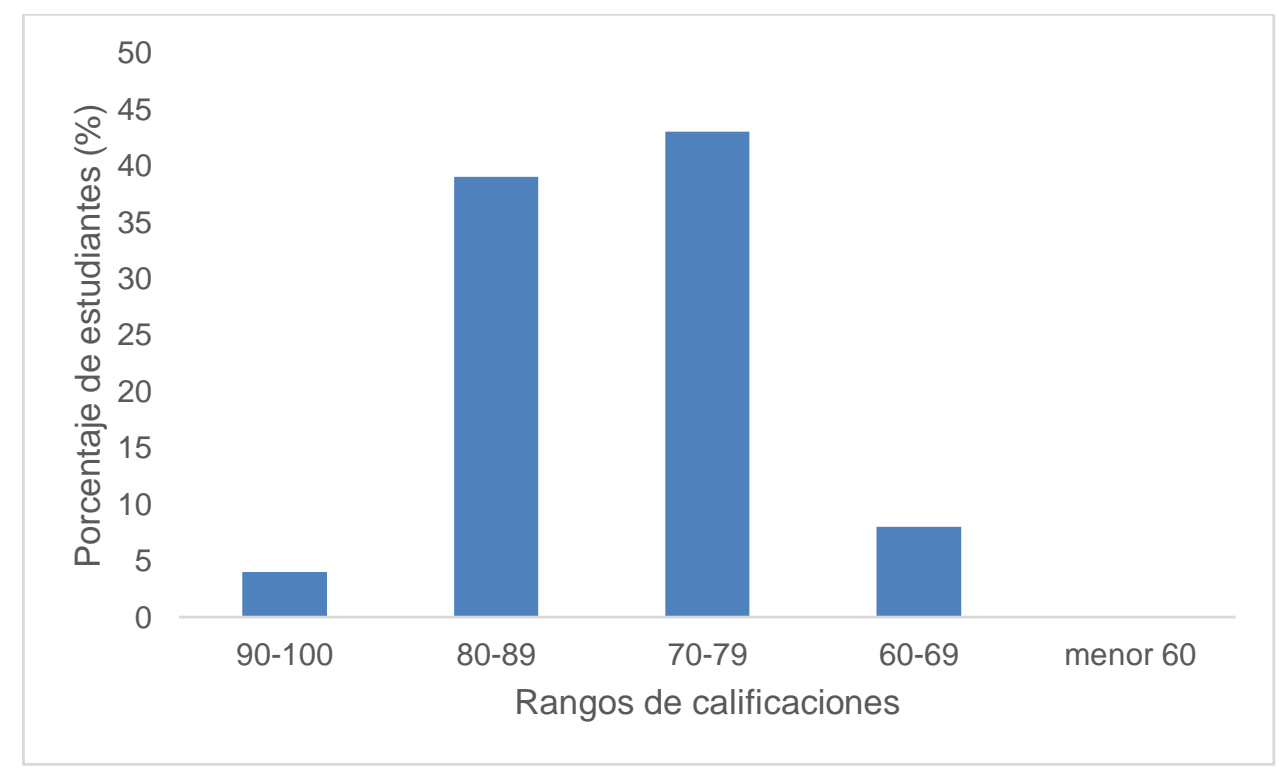

Fuente: Elaboración propia.

Estos resultados eran de esperar, ya que, el nivel académico exigido es mucho mayor en los colegios científicos que en las demás modalidades, debido a que, como parte de su

Evaluación del conocimiento básico en química en estudiantes de educación diversificada: El caso de Ingeniería Industrial, UNED.

Rodolfo Hernández-Chaverrí, Eric Montero-Miranda

Wendy Villalobos-González.

DOI : http://dx.doi.org/10.22458/caes.v8i1.1915 
visión principal, su enfoque está en brindar una educación sólida en las ciencias exactas como la química (Mata, 2011).

Seguidamente, el gráfico de la figura 2, refleja los resultados obtenidos por parte de los estudiantes que asisten a colegios públicos del MEP, y que representan al estudiante promedio de secundaria en Costa Rica a nivel de química.

Para este caso, se aplicó la prueba a una población de 102 estudiantes de colegios diurnos y nocturnos, obteniendo como resultado que cerca del $78 \%$ alcanzaron notas por debajo de 60 y un $22 \%$ lograron superar la nota de 70 .

Figura 2. Distribución del porcentaje de estudiantes de colegio públicos, según los rangos de calificaciones obtenidos, año 2014.

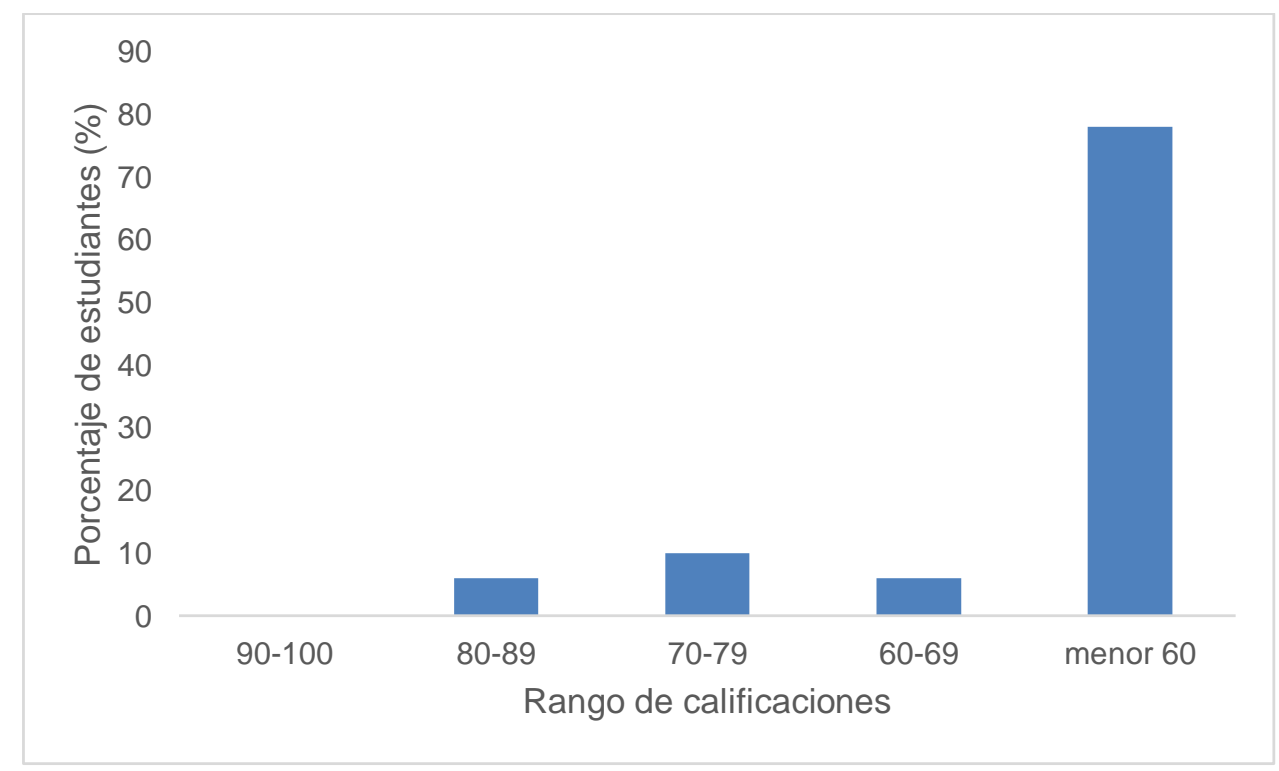

Fuente: Elaboración propia.

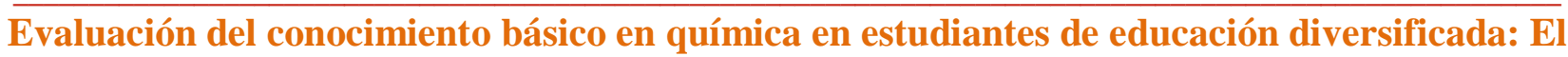
caso de Ingeniería Industrial, UNED.

Rodolfo Hernández-Chaverrí, Eric Montero-Miranda

Wendy Villalobos-González

DOI : http://dx.doi.org/10.22458/caes.v8i1.1915 


\subsection{Resultados obtenidos por los estudiantes aspirantes a la carrera de Ingeniería Industrial en la prueba diagnóstico de química para los años 2013 y 2014}

La prueba diagnóstica fue aplicada a la población aspirante a la carrera de Ingeniería Industrial. Para el año 2013, la prueba fue aplicada a 73 estudiantes aspirantes a la carrera de Ingeniería Industrial, en donde se observa una media aritmética de: 49. Además, cerca del $84 \%$ de los estudiantes obtuvieron notas por debajo de 60 , en contraste a lo observado en los estudiantes de colegios científicos en donde la mayoría se encontraba en rangos superiores a 70 .

Figura 3. Distribución del porcentaje de estudiantes aspirantes a la carrera de Ingeniería Industrial, según los rangos de calificaciones obtenidos para la prueba diagnóstico del año 2013.

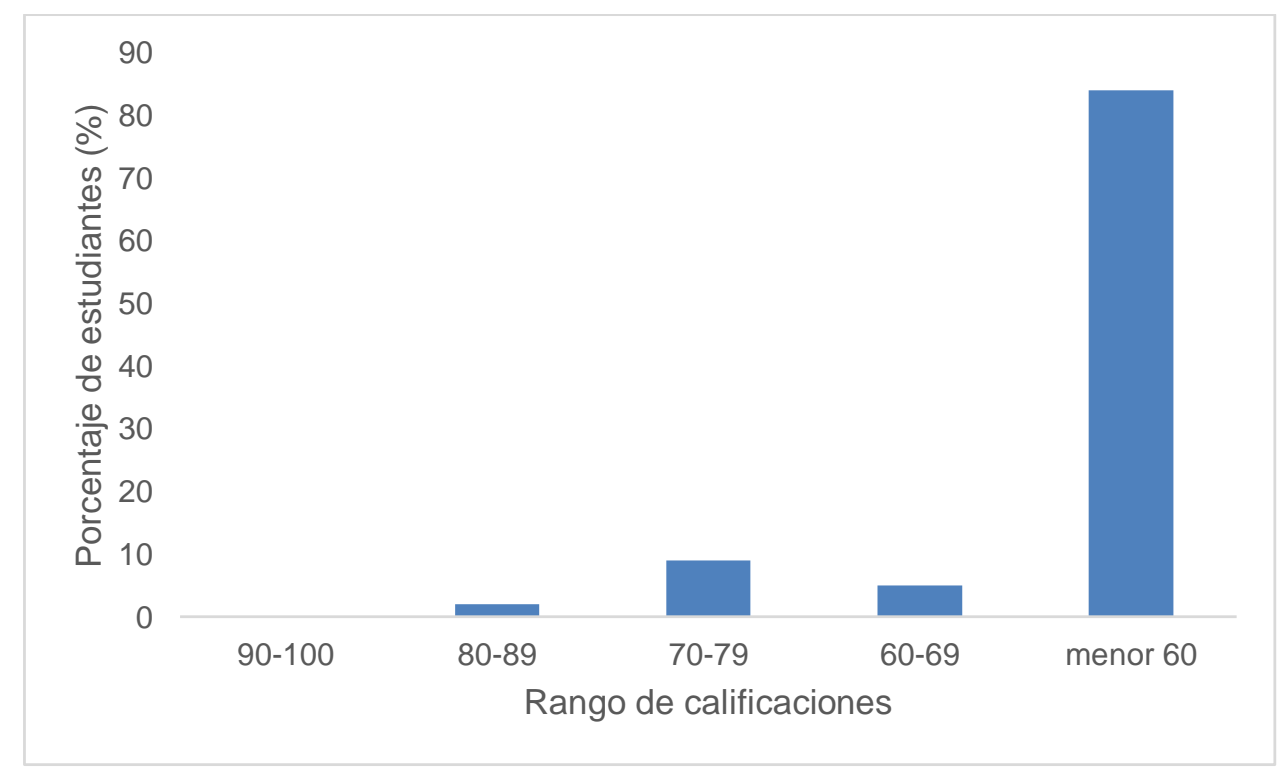

Fuente: Elaboración propia.

Evaluación del conocimiento básico en química en estudiantes de educación diversificada: El caso de Ingeniería Industrial, UNED.

Rodolfo Hernández-Chaverrí, Eric Montero-Miranda

Wendy Villalobos-González.

DOI : http://dx.doi.org/10.22458/caes.v8i1.1915 
Para el año 2014 se observa una tendencia muy similar a la del año 2013. En este caso, se aplicó la prueba a un total de 80 estudiantes aspirantes a la carrera de Ingeniería Industrial, en donde se observa una media aritmética de: 47 . Además, cerca del $83 \%$ de los estudiantes obtuvieron notas por debajo de 60 , además se contabilizó un $10 \%$ de estudiantes con notas superiores al 70 .

Figura 4. Distribución del porcentaje de estudiantes aspirantes a la carrera de Ingeniería Industrial, según los rangos de calificaciones obtenidos para la prueba diagnóstico del año 2014.

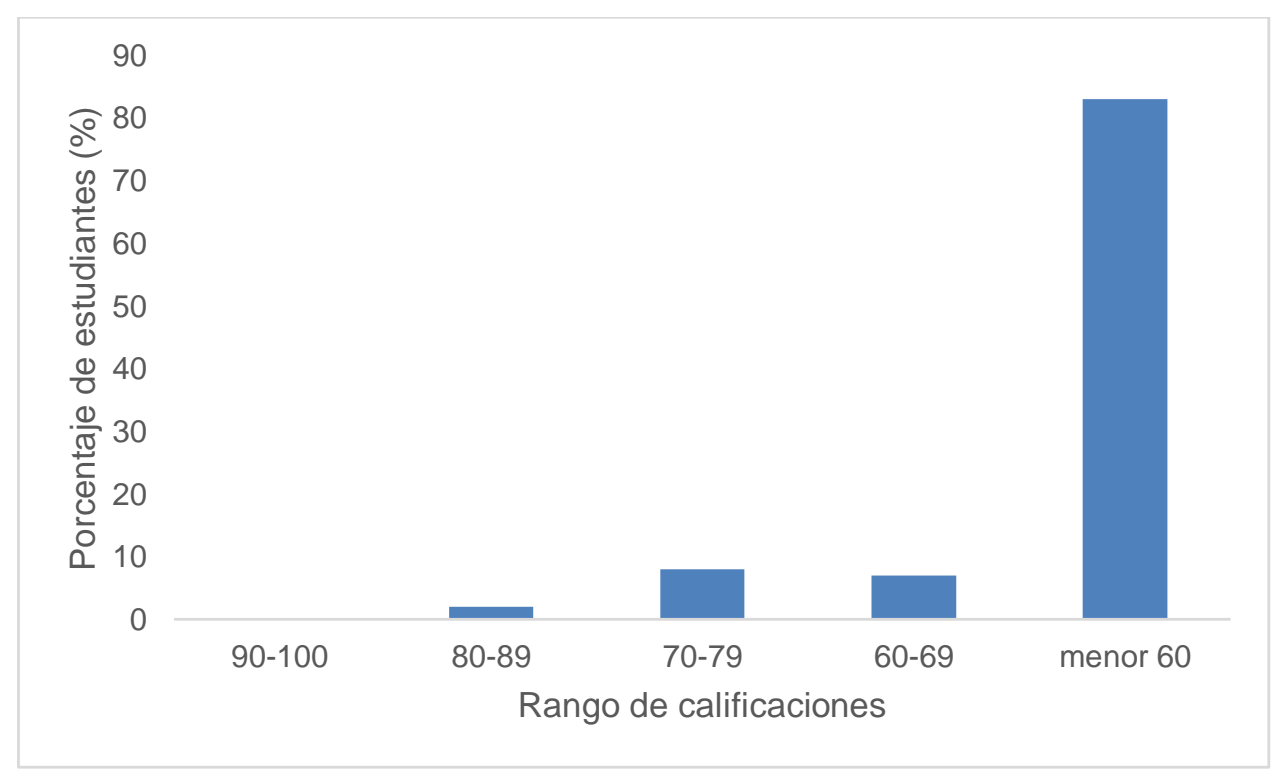

Fuente: Elaboración propia.

En un análisis general se sigue un patrón muy marcado entre los gráficos presentados en las figuras 2, 3 y 4, en donde se evidencia que la mayor población aspirante a carreras de la UNED es la que sale de colegios públicos, técnicos y algunos privados. Los estudiantes de colegios científicos marcan una diferencia muy considerable en cuanto al nivel académico, como se observó en la figura 1. Por lo tanto, es importante considerar el hecho de que el nivel académico para estudiantes de colegios científicos es superior a las otras

Evaluación del conocimiento básico en química en estudiantes de educación diversificada: El caso de Ingeniería Industrial, UNED.

Rodolfo Hernández-Chaverrí, Eric Montero-Miranda

Wendy Villalobos-González.

DOI : http://dx.doi.org/10.22458/caes.v8i1.1915 
modalidades, como los académicos, técnicos y privados. Además, existen una serie de factores que favorecen estos bajos rendimientos en la comunidad estudiantil que opta por ingresar a la carrera de ingeniería, y en general, a cualquier otra carrera que tenga como requisito cursar la asignatura de química.

En muchos casos los estudiantes solo llevaron la materia de biología o física, ya que, fue la asignatura que presentaron en sus pruebas de bachillerato y sus instituciones no resaltaron la importancia de las tres ciencias durante todo el ciclo diversificado, este caso es muy común en muchas instituciones privadas, en donde se enfocan en preparar a los estudiantes en su prueba de bachillerato según la ciencia seleccionada. De igual forma, gran parte de las instituciones de secundaria del Estado, enfocan la segunda mitad del año en preparar a sus estudiantes para las pruebas de bachillerato y se puede apreciar que los estudiantes de undécimo año bajan la carga académica en todas sus materias para dedicar más tiempo a repasar y estudiar para las pruebas de bachillerato. Históricamente, el mayor porcentaje de estudiantes optan por la biología para presentar la prueba de ciencias (cerca del $73 \%$ de los estudiantes según datos del MEP).

Otro fenómeno muy importante de tomar en cuenta es, que dada la modalidad y la oportunidad que brinda la UNED, la población que ingresa en su mayoría, son: 1) personas que están laborando desde hace años en alguna empresa y les solicitan en sus trabajos mejorar su nivel académico para optar por mejores opciones salariales y posiciones dentro de la empresa. 2) personas con bajos rendimientos académicos y bajos recursos. 3) personas de zonas muy alejadas.

Al analizar cada caso por separado se puede evidenciar que, en el primero, los estudiantes estuvieron un largo tiempo sin estudiar ninguna asignatura y con esto se incluye la química, por lo que el conocimiento de esta ciencia es deficiente. Para el segundo caso, muchos de

Evaluación del conocimiento básico en química en estudiantes de educación diversificada: El caso de Ingeniería Industrial, UNED. Rodolfo Hernández-Chaverrí, Eric Montero-Miranda

Wendy Villalobos-González.

DOI : http://dx.doi.org/10.22458/caes.v8i1.1915 
los estudiantes que obtuvieron bajos rendimientos en secundaria no pueden ingresar a otras universidades, en donde las carreras se manejan con examen de admisión y la nota ponderada de los últimos dos años del colegio. Dada la dificultad de la química, es probable que el nivel académico de esta ciencia también sea bajo. Así, a los bajos rendimientos se suma la situación económica, la que impide en muchos casos ingresar a una universidad privada. Como tercer punto, a muchos estudiantes se les dificulta el traslado al Valle Central para estudiar por lo que optan por la UNED que tiene más proyección en las comunidades alejadas del país, sin embargo, un estudio de Madrigal, Syedd, Montero y Vega en 2010, revela, que los estudiantes en zonas rurales poseen un nivel de conocimiento en química más bajo que en las zonas urbanas. Este trabajo revela que, de las instituciones participantes en la Olimpiada, cerca del $71 \%$ de los estudiantes que llegan a la prueba final pertenecen a instituciones ubicadas en zonas urbanas, y obtienen como media en la calificación final 48,42. Por otro lado, el 28,6\% de los estudiantes pertenecen a instituciones ubicadas en zonas rurales, y la media corresponde a 44,07.

Aunado a esto, se tiene también un nivel académico bajo debido a que en muchos casos el docente de la institución no posee el nivel de conocimiento actualizado de la materia a falta de capacitaciones.

\subsection{Resultados del curso de Química Nivelatoria}

Para el primer curso de Química Nivelatoria impartido durante I semestre del 2014, se matricularon un total de 120 estudiantes. En el gráfico 6, se observa que cerca del $44 \%$ de los estudiantes matriculados abandonaron el curso antes de realizar su primer examen parcial y del resto de estudiantes se determinó que cerca del 20,6\% de los estudiantes aprobaron el curso y el otro $79,4 \%$ reprobaron el mismo. En este último porcentaje se suman las deserciones que hubo tanto para el segundo como en el tercer examen parcial del curso.

Evaluación del conocimiento básico en química en estudiantes de educación diversificada: El caso de Ingeniería Industrial, UNED. Rodolfo Hernández-Chaverrí, Eric Montero-Miranda

Wendy Villalobos-González.

DOI : http://dx.doi.org/10.22458/caes.v8i1.1915 
Figura 5. Rendimientos académicos en el curso de Química Nivelatoria de los estudiantes aspirantes a la carrera de Ingeniería Industrial de la UNED en el I semestre, 2014.

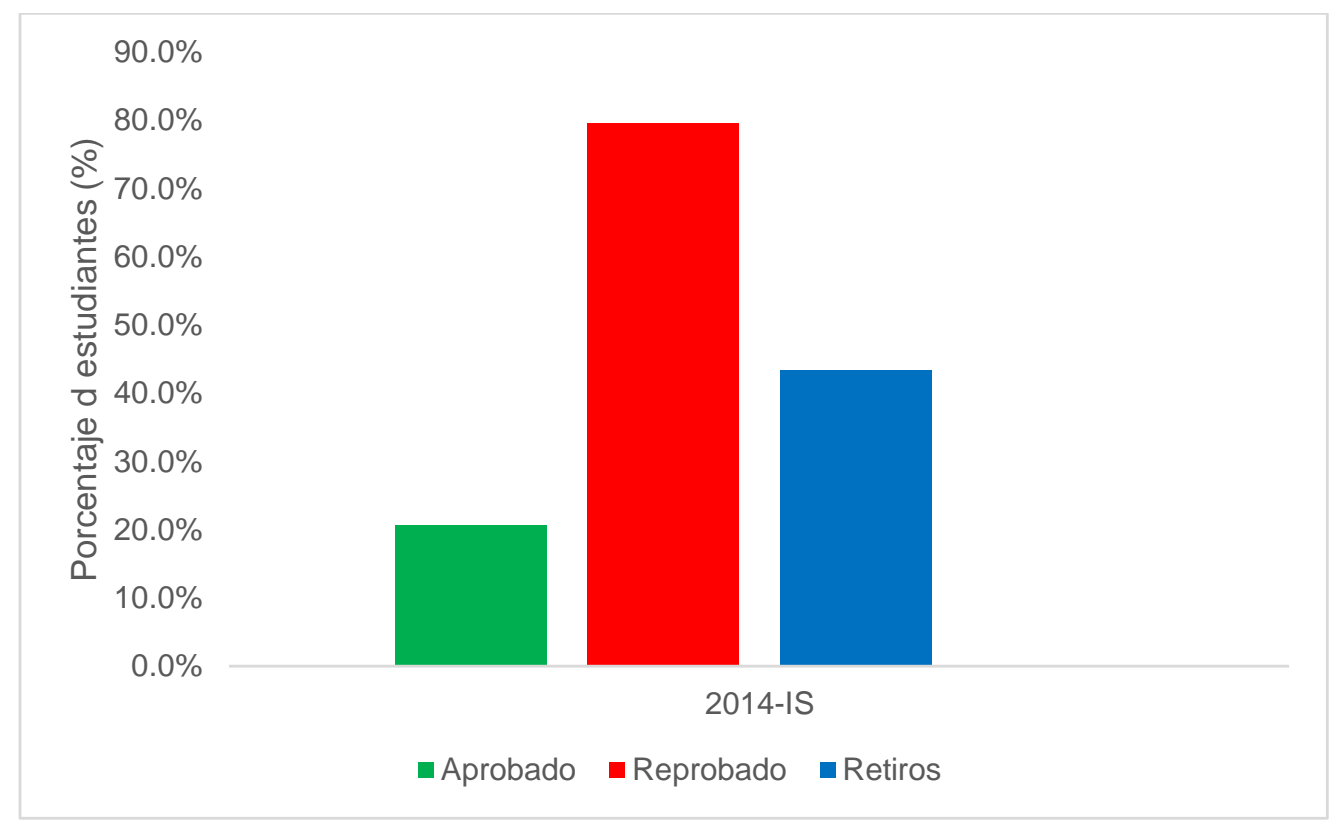

Fuente: Elaboración propia.

A partir del segundo semestre 2014, la Cátedra de Ciencias Químicas decide realizar cambios sustanciales en el modelo de evaluación del curso, con el fin de mejorar la promoción académica de los estudiantes que matriculan el curso de Química Nivelatoria. Dichas modificaciones se exhiben en la siguiente tabla:

Evaluación del conocimiento básico en química en estudiantes de educación diversificada: El caso de Ingeniería Industrial, UNED. Rodolfo Hernández-Chaverrí, Eric Montero-Miranda

Wendy Villalobos-González

DOI : http://dx.doi.org/10.22458/caes.v8i1.1915 
Tabla 1. Modelos de evaluación aplicados para la asignatura de Química Nivelatoria en el I y |l semestre, 2014.

\begin{tabular}{|c|c|}
\hline $\begin{array}{l}\text { Modelo de evaluación I semestre } \\
\qquad 2014\end{array}$ & $\begin{array}{l}\text { Modelo de evaluación II semestre } \\
2014\end{array}$ \\
\hline $\begin{array}{l}5 \text { pruebas cortas en línea } \\
2 \text { tareas en línea } \\
3 \text { pruebas ordinarias presenciales }\end{array}$ & $\begin{array}{l}5 \text { pruebas cortas en línea } \\
2 \text { tareas en línea } \\
3 \text { pruebas ordinarias en línea* } \\
2 \text { pruebas ordinarias presenciales* }\end{array}$ \\
\hline
\end{tabular}

Fuente: Cátedra de Ciencias Químicas, UNED.

* De las tres pruebas ordinarias planteadas originalmente, la I y III se dividió en dos partes, en este caso, el enfoque teórico se adecuó en una prueba en línea y el enfoque de resolución de problemas se adecuó a una prueba presencial. El caso del II ordinario, se realizó una única prueba en línea.

Una de las premisas para la modificación en el modelo evaluativo es que el estudiante, tenga mayor opción de aprobar el curso, ya que, al tener evaluaciones en el hogar, puede autorregularse de tal forma que se ve obligado a repasar los tópicos evaluados para responder la prueba, además de tener la opción de dos días y dos intentos para realizar la prueba en línea a la hora más conveniente para el estudiante.

Para el segundo curso desarrollado en la segunda mitad del año, matricularon un total de 38 estudiantes de los cuales, como lo refleja el grafico de la figura 6 , el $41 \%$ desertó el curso sin haberlo comenzado, del restante porcentaje, que sí continuó, un 30\% aprobó la asignatura en tanto el otro $70 \%$ reprobó el curso.

Evaluación del conocimiento básico en química en estudiantes de educación diversificada: El caso de Ingeniería Industrial, UNED. Rodolfo Hernández-Chaverrí, Eric Montero-Miranda

Wendy Villalobos-González

DOI : http://dx.doi.org/10.22458/caes.v8i1.1915 
Figura 6. Rendimientos académicos en el curso de Química Nivelatoria de los estudiantes aspirantes a la carrera de Ingeniería Industrial de la UNED en el II semestre, 2014.

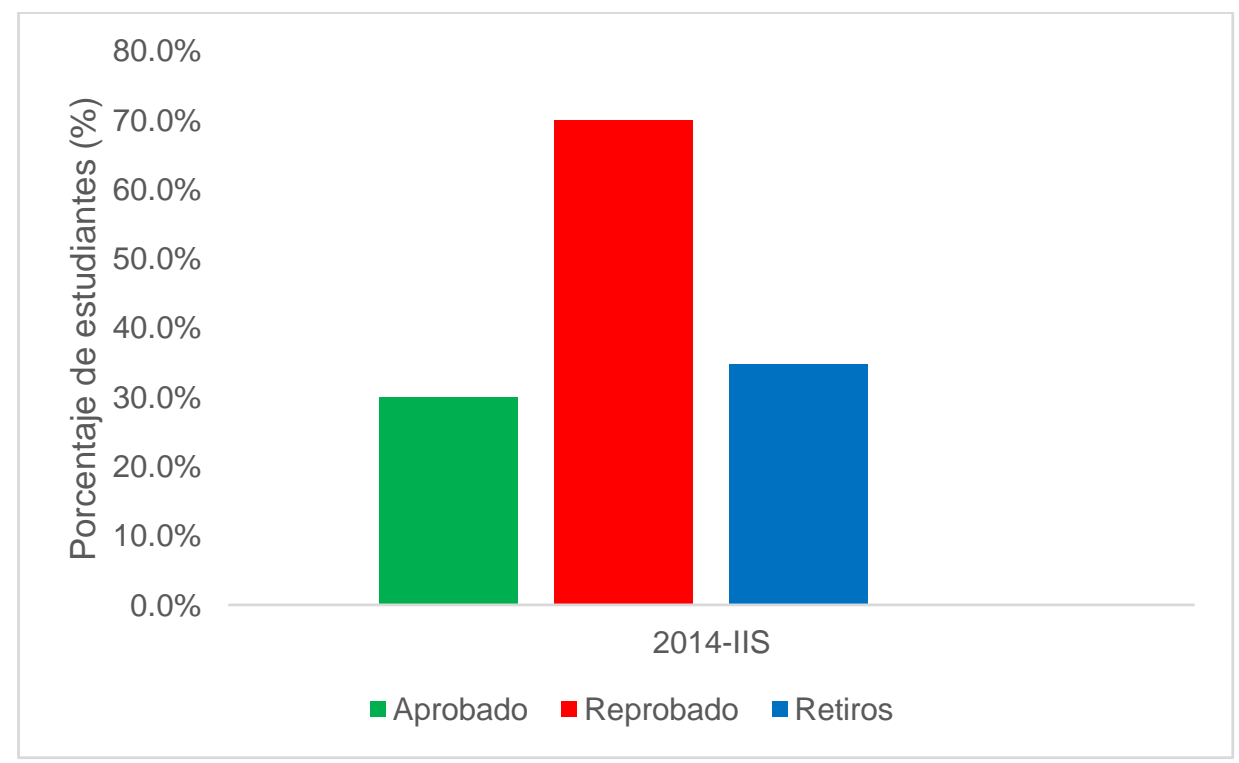

Fuente: Elaboración propia.

Al realizar una comparación de los resultados obtenidos en el I y II semestre, se puede notar que respecto al I semestre, en el segundo, se tiene una mejoría notoria en cuanto a los porcentajes de estudiantes que aprobaron el curso (pasando de un $20,6 \%$ en el I semestre a un $30 \%$ en el II semestre).

Evaluación del conocimiento básico en química en estudiantes de educación diversificada: El caso de Ingeniería Industrial, UNED. 
En la figura 7, se muestra un gráfico de la cantidad de estudiantes aprobados y reprobados del curso de Química Nivelatoria desde el II semestre 2014 hasta el II semestre 2016.

Figura 7. Rendimiento académico de los estudiantes de Química Nivelatoria periodo II semestre 2014 a II semestre 2016.

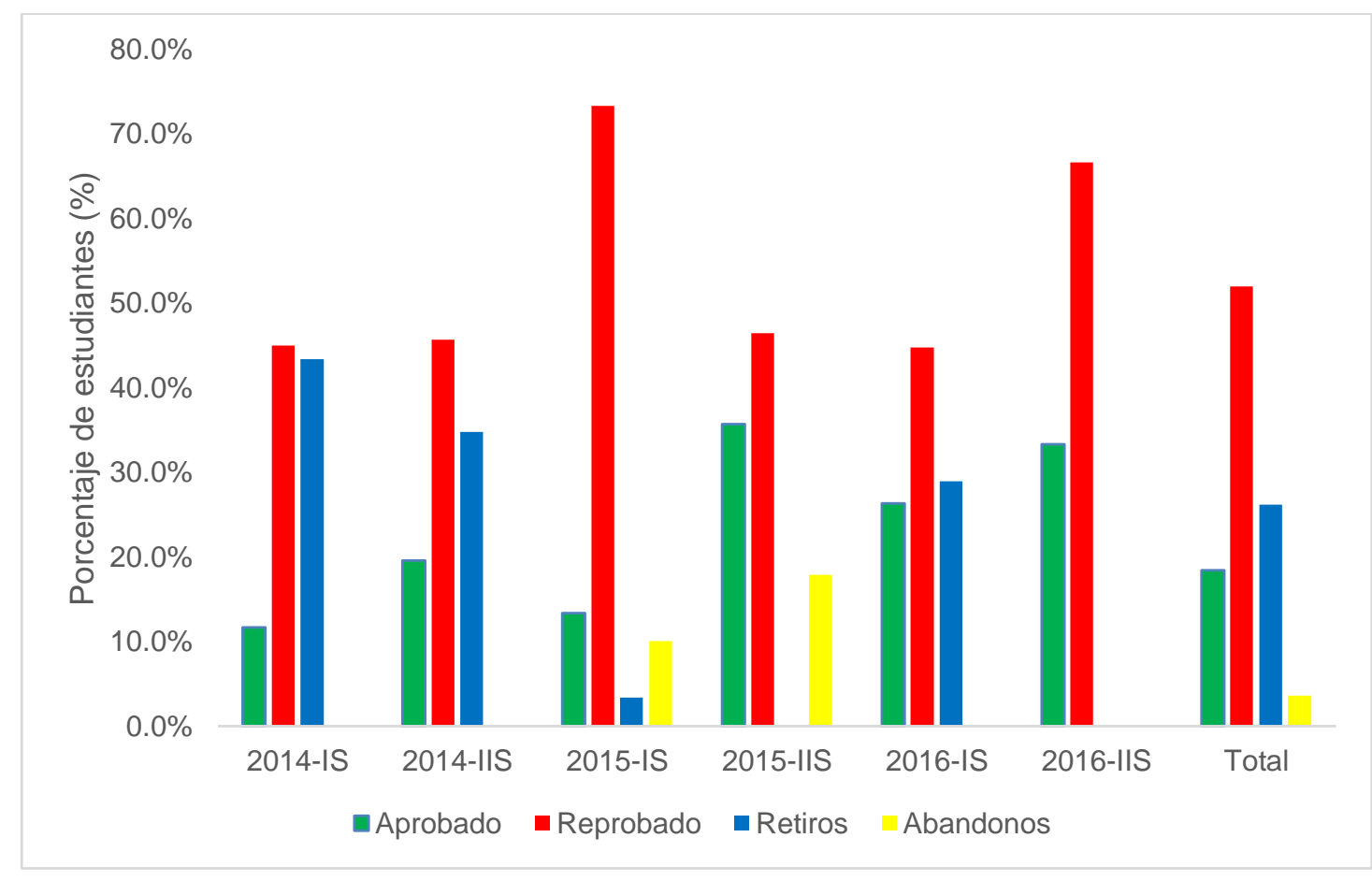

Fuente: Elaboración propia.

\subsection{Comparación entre el rendimiento académico de los estudiantes que cursan} Química I (cuatrimestral) de la UNED y los estudiantes de ingeniería Industrial

Como ya se ha mencionado con anterioridad, los estudiantes de Ingeniería industrial antes de llevar el curso de Química I deben aprobar el curso de Química Nivelatoria. En la figura

Evaluación del conocimiento básico en química en estudiantes de educación diversificada: El caso de Ingeniería Industrial, UNED.

Rodolfo Hernández-Chaverrí, Eric Montero-Miranda

Wendy Villalobos-González.

DOI : http://dx.doi.org/10.22458/caes.v8i1.1915 
8, se muestra el rendimiento académico del curso de Química I cuatrimestral desde el año 2015 al 2016.

Figura 8. Rendimientos académicos en el curso de Química I de los estudiantes regulares de la UNED en el periodo III cuatrimestre 2015-2016.

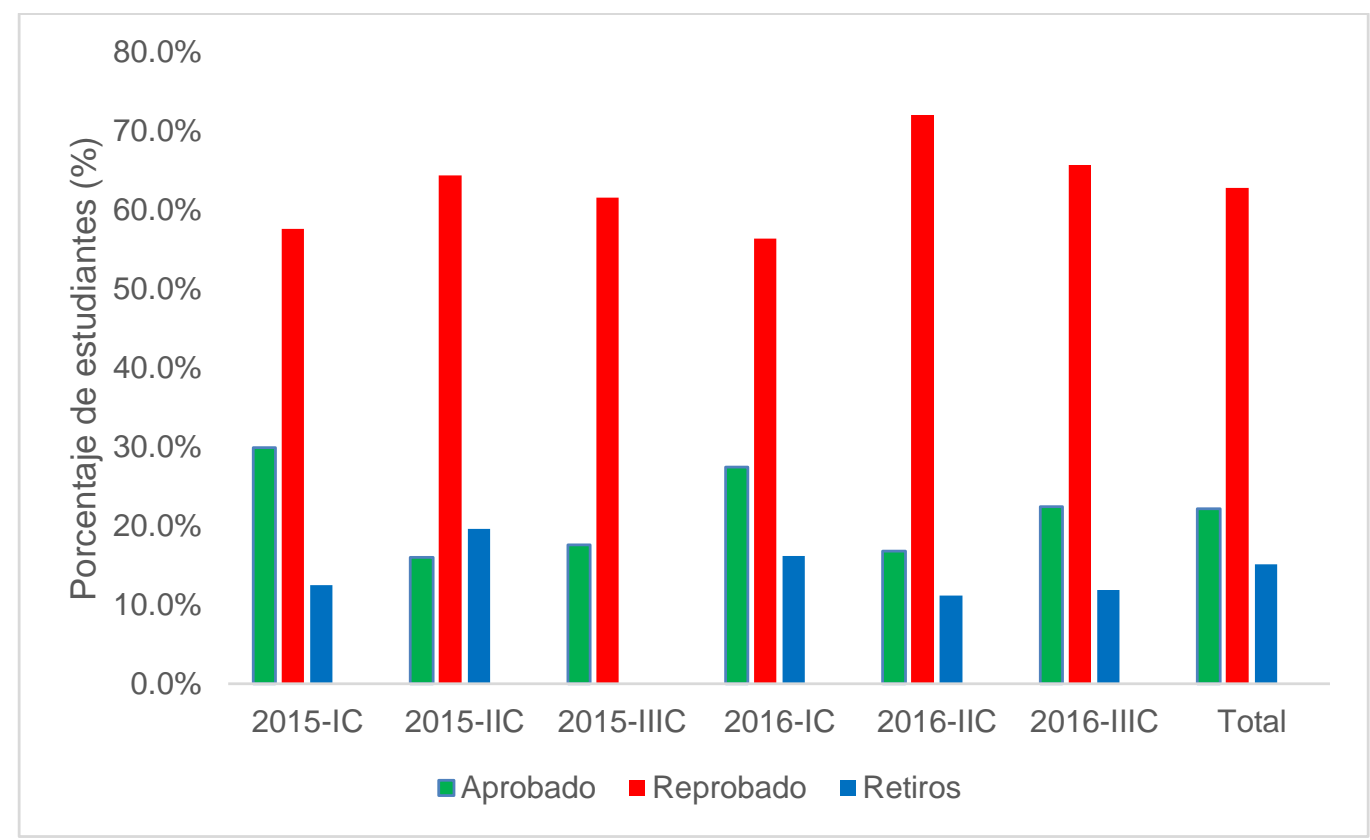

Fuente: Elaboración propia.

Todos los factores de bajo rendimiento discutidos en el punto 3.2 se ven reflejadas en el gráfico 3, que exhibe los porcentajes de estudiantes que aprueban, pierden y retiran el curso de Química I, impartido por la UNED para otras carreras durante el periodo 20152016. En dicho gráfico se observa que el porcentaje estudiantes que pierden el curso de Química I, duplica y hasta triplica el porcentaje de estudiantes que aprueban el curso. En un análisis global de estas tendencias se observa un $62,7 \%$ de estudiantes que pierden el curso contra un $22,1 \%$ que lo aprueba, el otro $15,1 \%$ retira el curso, lo que representaría

Evaluación del conocimiento básico en química en estudiantes de educación diversificada: El caso de Ingeniería Industrial, UNED.

Rodolfo Hernández-Chaverrí, Eric Montero-Miranda

Wendy Villalobos-González

DOI : http://dx.doi.org/10.22458/caes.v8i1.1915 
un $77,8 \%$ de los estudiantes que pierden este curso. En el gráfico de la figura 9 , se muestra el rendimiento académico de los estudiantes de Ingeniería Industrial en Química General I semestral durante el periodo 2015-2016.

Figura 9. Rendimiento académico de los estudiantes de Ingeniería Industrial en Química General I.

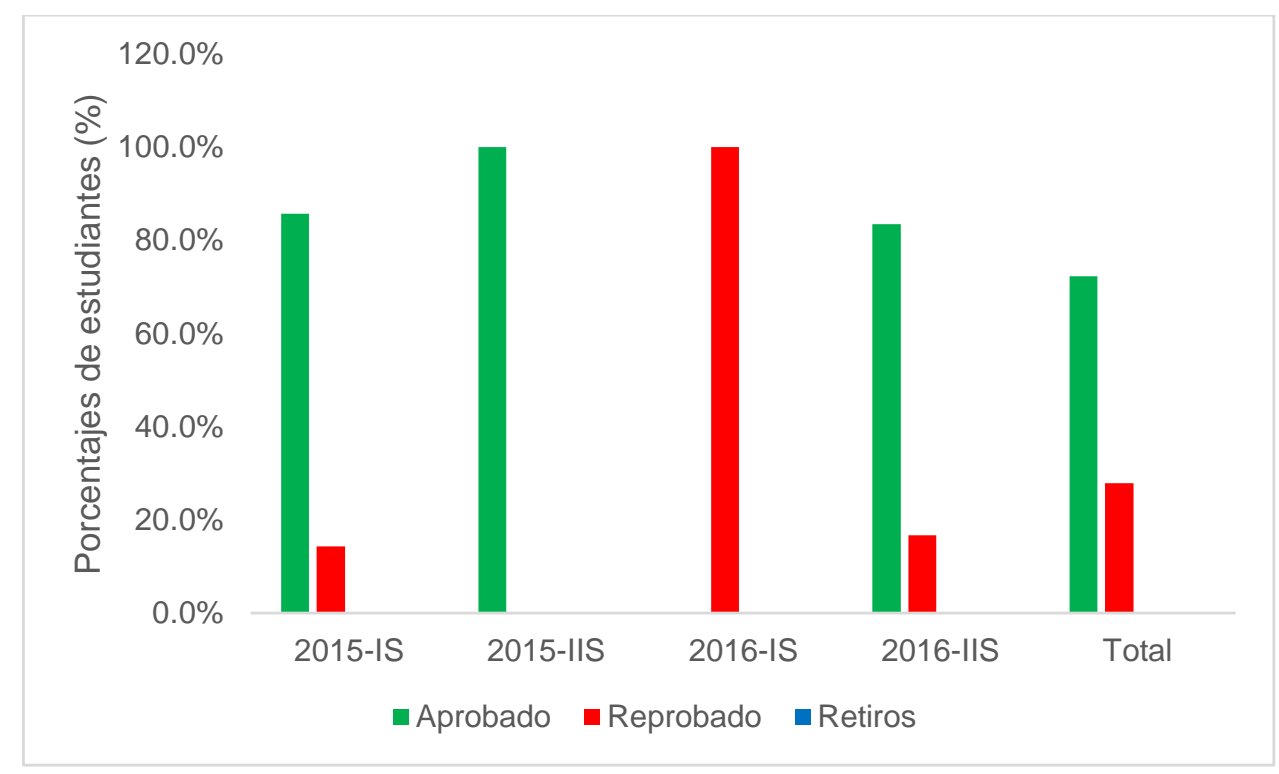

Fuente: Elaboración propia.

En el gráfico de la figura 9, se muestra cómo en total el $72,2 \%$ de los estudiantes aprueban el curso de Química I y un 27,8\% lo reprueba. Este contraste observado entre ambos tipos de cursos, que son totalmente iguales en contenido, puede ser atribuido a dos variables. La primera, los estudiantes de Química General I semestral deben aprobar antes el curso de Química Nivelatoria, por lo que tienen los contenidos de secundaria más recientes.

La otra variable importante es el tiempo de asimilación de contenidos, es decir, el paso del curso de modo cuatrimestral a semestral. En el curso semestral se cuenta con tres pruebas

Evaluación del conocimiento básico en química en estudiantes de educación diversificada: El caso de Ingeniería Industrial, UNED.

Rodolfo Hernández-Chaverrí, Eric Montero-Miranda

Wendy Villalobos-González.

DOI : http://dx.doi.org/10.22458/caes.v8i1.1915 
ordinarias y el contenido del mismo se va integrando en forma más dosificada por el factor tiempo. Mientras que en el curso cuatrimestral se tienen dos pruebas ordinarias y los contenidos se dan con una mayor dosis por tiempo de contacto, el cual es menor. El modelo de evaluación de estos dos cursos se muestra en la tabla 2.

Tabla 2. Modelos de evaluación aplicados para la asignatura de Química I semestral y Química I cuatrimestral.

\begin{tabular}{|c|c|}
\hline $\begin{array}{l}\text { Modelo de evaluación Química I } \\
\text { semestral }\end{array}$ & $\begin{array}{c}\text { Modelo de evaluación Química I } \\
\text { cuatrimestral }\end{array}$ \\
\hline 6 pruebas cortas en línea $20 \%(3.33 \%$ & 4 pruebas cortas en línea $20 \%$ (5\% c/u) \\
\hline 2 tareas en línea $20 \%(10 \% \mathrm{c} / \mathrm{u})$ & 2 tareas en lín \\
\hline $\begin{array}{l}3 \text { pruebas ordinarias presenciales } 60 \% \\
(20 \% \mathrm{c} / \mathrm{u})\end{array}$ & $\begin{array}{l}2 \text { pruebas ordinarias presenciales } 60 \% \\
(30 \% \mathrm{c} / \mathrm{u})\end{array}$ \\
\hline
\end{tabular}

Esto deja en evidencia de que un curso previo, que ayude al estudiante a nivelar su conocimiento en esta ciencia, puede influir de forma importante en la mejora de los cursos de química propios de la malla curricular de las carreras ofrecidas por la UNED, y de forma más puntual en la carrera de Ingeniería Industrial.

\section{CONCLUSIONES}

Como parte de los aprendizajes extraídos de esta experiencia, se concluye que la elaboración, validación y aplicación de una prueba diagnóstico en el área de química, es una herramienta útil para poder segregar de una forma más adecuada la población de estudiantes que desean ingresar a la carrera de Ingeniería Industrial, y que en dado momento presentan un nivel bajo de conocimiento en esta ciencia. Por otro lado, esta

Evaluación del conocimiento básico en química en estudiantes de educación diversificada: El caso de Ingeniería Industrial, UNED.

Rodolfo Hernández-Chaverrí, Eric Montero-Miranda

Wendy Villalobos-González

DOI : http://dx.doi.org/10.22458/caes.v8i1.1915 
prueba brinda información importante acerca de los temas y contenidos donde existen deficiencias en la asignatura de química.

El curso Química Nivelatoria es una herramienta útil para solventar las deficiencias que los estudiantes arrastran desde la secundaria, y así, poder mejorar su rendimiento académico en los cursos de química que son parte del plan de estudios de la carrera de Ingeniería Industrial.

El diseño del curso de Química Nivelatoria está enfocado en la solvencia de las carencias académicas que fueron expuestas por los resultados arrojados de la aplicación del examen diagnóstico. Ambas herramientas son complementarias y de gran importancia para el planteamiento de estrategias enfocadas a la mejora de la enseñanza de la química a nivel de educación superior.

Tanto la aplicación de la prueba diagnóstico como los primeros resultados obtenidos en el curso de Química Nivelatoria, revelan que los niveles académicos en el área de química son bajos y deficientes en comparación con los colegios científicos, en donde las promociones obtenidas en la prueba diagnostico son altas.

El curso de Química General I semestral presenta un mayor porcentaje de aprobación (72,1\%) que el curso de Química I cuatrimestral, esto se presume a partir del efecto que tiene el curso de Química Nivelatoria previo que llevan estos estudiantes. Sin embargo, es muy pronto indicar que la mejor opción es la que se da en modo semestral, dado que la población analizada es muy pequeña en comparación con la del modo cuatrimestral.

Evaluación del conocimiento básico en química en estudiantes de educación diversificada: El caso de Ingeniería Industrial, UNED. Rodolfo Hernández-Chaverrí, Eric Montero-Miranda

Wendy Villalobos-González.

DOI : http://dx.doi.org/10.22458/caes.v8i1.1915 


\section{RECOMENDACIONES}

Como parte de la experiencia recabada por la Cátedra de Ciencias Químicas, se recomienda que este pilotaje sea exportado a otras carreras de la universidad, ya que, estos procesos evaluativos evidencian en este caso las carencias que los estudiantes arrastran desde la secundaria en diferentes asignaturas y permite a cada instancia establecer acciones correctivas y de mejora.

La Escuela de Ciencias Exactas y Naturales ha sido una instancia que se ha preocupado por brindar las herramientas y medios necesarios para que otras cátedras desarrollen actividades similares a la de Química, sin embargo, se recomienda que invierta más recursos humanos y económicos para poder trasladar esta experiencia a otras carreras. La Universidad Estatal a Distancia debe trabajar en la mejora de esta problemática desde su origen, es decir, solventar este problema en la medida de lo posible desde la secundaria mediante la capacitación de docentes del área de ciencias al menos dos veces al año en temas que presenten mayor deficiencia.

Es necesario que el Ministerio de Educación Pública doble esfuerzos para asegurar una mayor calidad y fiscalización de la educación que reciben los jóvenes costarricenses, y así evitar estas deficiencias tan marcadas a la hora de ingresar a carreras universitarias, para ello se insta a la coordinación de instituciones como la UNED para establecer estrategias de mejora.

Es importante realizar una valoración a futuro exponiendo a los estudiantes a un curso nivelatorio o introductorio previo a matricular la asignatura cuatrimestral, o realizar un movimiento institucional para que en el caso de Química General se oferte en modo semestral a todas las carreras que lo solicitan como curso de servicio.

Evaluación del conocimiento básico en química en estudiantes de educación diversificada: El caso de Ingeniería Industrial, UNED. Rodolfo Hernández-Chaverrí, Eric Montero-Miranda

Wendy Villalobos-González.

DOI : http://dx.doi.org/10.22458/caes.v8i1.1915 


\section{REFERENCIAS}

Colegio Científico Costarricense de San Pedro. (2014). Admisión. Recuperado de http://colegiocientificosanpedro.ed.cr/node/2

Ley de Promoción del Desarrollo Científico y Tecnológico de 1990. La Gaceta 7169.

Ley Fundamental de Educación de 1957. La Gaceta § 2160.

Madrigal, A., Syedd, R., Montero, E., \& Vega, J. (2010). Análisis de las Olimpiadas Nacionales de Química en Costa Rica: Alcances y retos para la población joven. UNICIENCIA, 26(1), 29-40.

Marín, M. G. (2010). Antología de calidad (2da ed.), San José, Costa Rica: Ministerio de Educación Pública.

Mata, S. (2011). Colegios Científicos de Costa Rica se convierten en ascensores para movilidad social del país. Informa TEC, 315 . Recuperado de http://www.tec.cr/prensa/informatec/2011/agosto/n8.html

Programa de química para bachillerato (s.f.). San José, Costa Rica: Ministerio de Educación Pública.

Ramírez, J. L. (2006). Las tecnologías de la información y de la comunicación en la educación en cuatro países latinoamericanos. Revista Mexicana de Investigación Educativa, 11(28), 61-90

Roldán, L. (2012, 26 de octubre). Razones del éxito de los colegios científicos, La Nación. Recuperado de http://www.nacion.com/opinion/foros/Razones-exito-colegioscientificos_0_1301469884.html

Sol, R. (2004). Diagnóstico sobre la Formación de Docentes en Instituciones de Educación Superior en Costa Rica. San José, Costa Rica.

Tedesco, J. C., López, N. (2002). Desafíos a la educación secundaria en América Latina. Revista de la CEPAL, 76, 55-69.

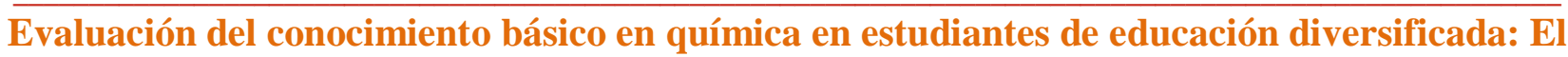
caso de Ingeniería Industrial, UNED.

Rodolfo Hernández-Chaverrí, Eric Montero-Miranda

Wendy Villalobos-González.

DOI : http://dx.doi.org/10.22458/caes.v8i1.1915 